\title{
La teología producida en la UCA
}

Rafael de Sivatte S.J.

Universidad Centroamericana José Simeón Cañas

\section{Teología y UCA caminan juntas}

Casi desde el principio de la existencia de la UCA, esta universidad y la teología han caminado a la par. Una teología académica y bien fundamentada pero cuyo punto de partida es la realidad, una teología que se ha querido mantener fiel a dicha realidad y que se ha intentado historizar continuamente; una teología, por otra parte, que intenta responder con una visión profundamente jesuítica, cristiana y liberadora a dicha realidad. De ahí que se hable de inspiración cristiana de la UCA y de universidad para el cambio social. Esta inspiración cristiana tuvo su historización a partir del año 1977 en la figura de monseñor Romero, quien dio su vida por transformar la sociedad en algo más justo y solidario para los empobrecidos y, además, fue y sigue siendo el espíritu que da vida a la misión de la UCA y a nuestra teología.

Como ya se sabe y puede deducirse de lo mencionado anteriormente, sobre todo en los primeros años de su existencia, los de la agudización de la conflictividad en El Salvador, la teología de la UCA en especial, y con ella la UCA entera, sufrió ataques, difamaciones, persecución, asesinatos de estudiantes y profesores; todo ello posiblemente por su preocupación por la realidad y por su ocupación en la transformación de la misma a la luz de la inspiración cristiana.

Parece cierto, pues, que la persecución general a la UCA ha sido debida en mucha parte a su producto teológico y a su tipo de profesorado. Todo esto culminó con el martirio de los seis jesuitas, Julia Elba y Celina el 16 de noviembre de 1989. De ahí la importancia que se ha dado y se da en nuestra teología a la tradición martirial, es decir testimonial, de la Iglesia salvadoreña, a la conservación de su memoria y a la reflexión y profundización de su significado y alcances.

No se debe dejar de mencionar que por su talante liberador y su compromiso en favor del cambio social de nuestra realidad tan injusta y desigual de la sociedad 
civil y eclesial, la teología y los teólogos de la UCA han sido vistos con frecuencia de un modo sospechoso y no han sido comprendidos, incluso por parte de la Iglesia y de su jerarquía.

\section{Teología y funciones de la UCA}

Como es conocido, la UCA tiene tres funciones constitutivas de su misión, totalmente interrelacionadas: la investigación, la docencia y la proyección social. Pues bien, como se verá a continuación; el Departamento de Teología ha integrado y quiere seguir integrando estas tres funciones.

\subsection{Docencia}

Quizá la dimensión más visible, y también cuantificable, de la labor teológica de la UCA es la docencia en diversos niveles: maestría, licenciatura, profesorado, escuelas de teología y cursos sistemáticos de diplomado.

La docencia formal de Teología comenzó en 1974 sobre todo con Ignacio Ellacuría y Jon Sobrino, con un programa de maestría, que no se pudo concluir a raíz del martirio de Rutilio Grande el año de 1977. Se reanudó en 1980, pero tampoco se pudo terminar debido al estallido de la guerra. Se volvió a comenzar como Maestría en Teología en el año de 1985 y se mantuvo con este nombre hasta el 2001.

El actual programa de Maestría en Teología Latinoamericana, iniciado el año 2012, cuenta ya con dos promociones y una docena de estudiantes por curso, entre los que hay clérigos y laicos de diversas nacionalidades y credos religiosos.

La Licenciatura en Teología, existente con este nombre desde 1997, tiene una matrícula de unos 125 estudiantes, la gran mayoría seminaristas de muchas de las familias religiosas de la región mesoamericana y de Suramérica y, por lo tanto, de muy diversas nacionalidades. El programa ha graduado desde 1999 hasta el presente a unos 375 estudiantes.

El Profesorado en Teología, fundado en 1983, tiene una matrícula que oscila entre 30 y 35 estudiantes, la mayoría de ellos, religiosas y religiosos destinados a enseñar religión y valores en colegios católicos, además, hay laicos y laicas al servicio de la comunidad cristiana como profesores de religión, catequistas o agentes de pastoral. Desde 1998 han recibido el título de Profesorado en Teología unos 200 estudiantes.

Las Escuelas de Teología Pastoral, fundadas en 1995, han tenido en promedio una matrícula de unos 600 estudiantes anuales, hombres y mujeres, de 
los cuales aproximadamente la mitad concluye el programa en los tres años previstos. El programa se imparte en una docena de centros, distribuidos en todo el país, pero la mayoría en el gran San Salvador.

Finalmente, están los cursos libres de Teología que fueron abiertos en 2010. Equivalen a un diplomado de quince horas y tienen una matrícula de unos 30 estudiantes por curso. Su organización depende de la Pastoral Universitaria.

Quiero acabar este apartado de la docencia haciendo notar que la docencia de Teología en estos niveles tan variados es impartida por profesores y profesoras especializados en las diversas ramas de la disciplina. Aunque la Teología siempre ha contado con un núcleo docente en la UCA, también ha contado, desde el comienzo, con la colaboración de teólogos y teólogas europeos, sobre todo españoles, norteamericanos y también suramericanos; todos ellos altamente cualificados por sus estudios, investigaciones y prestigio en el mundo de la Teología. La venida de profesores y profesoras de España, Alemania, Austria, Estados Unidos y América Latina, todos ellos y ellas muy sensibles a nuestra realidad y a nuestra teología, son una muestra de la solidaridad internacional con nuestra teología liberadora. A lo largo de los últimos 30 años se suman unos 20, entre doctores y doctoras en Teología.

\subsection{Investigación}

Ahora bien, la calidad de la docencia de Teología depende directamente de las investigaciones de sus docentes. Es verdad que esta actividad investigadora, en buena medida por la naturaleza de la disciplina, es en el caso de la Teología menos tangible y más conocida en el exterior que en el mismo El Salvador, ya que la transmisión se hace por medio de la docencia a grupos reducidos de estudiantes de Teología y por revistas especializadas poco extendidas en nuestro país. Sin embargo, ella es muy real.

\subsection{Proyección social}

Cabe destacar en este sentido que, de una u otra manera, el saber teológico y la reflexión teológica desde la realidad salvadoreña y centroamericana pasan, a partir de nuestras carreras, a quienes están al servicio de las comunidades; a las profesoras y profesores de religión de colegios y escuelas diversas, y a los agentes de pastoral dispersos por las parroquias del país. Asimismo, conviene destacar que ese saber y esa reflexión también llegan a las comunidades salvadoreñas a través de la radio YSUCA, publicaciones como Carta a las Iglesias, cuyo primer número es de 1981, los 33 cuadernos de divulgación teológica y pastoral que desde 1998 publica el Centro Monseñor Romero, las 18 cartillas de las Escuelas de 
Teología Pastoral, las conferencias, los talleres, las charlas, entre otros.

También se puede decir que dicho saber y reflexión teológica desde la realidad salvadoreña y centroamericana ha llegado y sigue llegando a centenares de estudiantes del país, de toda el área centroamericana y de otros países latinoamericanos. Estos estudiantes, provenientes de varias congregaciones religiosas y en creciente número del mundo laico, al regresar a sus ambientes naturales y a sus países prolongan el espíritu de nuestra teología.
Así pues, la investigación y la reflexión teológica desde la realidad histórica poseen una destacada proyección social, a través de los diversos niveles de la docencia, la publicación de libros y artículos en revistas especializadas y de divulgación, las conferencias, los talleres, las charlas, los cursillos y la participación en diversos foros y comités internacionales. Estas actividades forman parte de la proyección social del Departamento $y$, en último término, de la UCA como un todo.

\section{Publicaciones}

\subsection{De los iniciadores de esta teología de la UCA}

No voy ahora a hacer un listado, uno por uno, de los títulos de los libros, artículos y folletos de cada miembro del Departamento, que son fruto de la docencia y de la investigación. Eso sería interminable y tedioso. Lo que sí quiero presentar son las grandes temáticas investigadas y publicadas por los diferentes miembros del equipo teológico de la UCA, mostrando cómo la teología de la UCA ha iluminado las realidades conflictivas o esperanzadoras de cada momento. En realidad, por el Stand colocado en el vestíbulo puede hacerse una idea de la cantidad y calidad de la investigación y publicaciones del Departamento
Pero en este apartado lo primero que conviene resaltar es el conjunto de las obras publicadas, fruto de pequeñas o grandes investigaciones, de Ignacio Ellacuría y Jon Sobrino, iniciadores de toda esta línea teológica en El Salvador. Muchos de estos escritos han sido traducidos a diferentes idiomas como inglés, portugués, francés, italiano, alemán, y alguno de ellos al croata y al coreano.

No se puede decir que de Ellacuría tengamos ya las obras completas publicadas, pues todavía quedan muchos textos inéditos en el archivo que lleva su nombre, declarado Patrimonio Cultural de la Humanidad, y que actualmente está en línea. Pero está claro que sí están publicados los conjuntos 
más importantes de sus escritos: los tres volúmenes de sus Escritos Filosóficos, los cuatro de sus Escritos Teológicos, los tres de sus Escritos Políticos (titulados Veinte años de historia en El Salvador 1969-1989), el de los Escritos Universitarios y la Filosofía de la Realidad Histórica.

En el caso de Jon Sobrino, destacan sus dos obras mayores sobre Cristología: Jesucristo Liberador. Lectura histórica-teológica de Jesús de Nazaret (1991) con 455 páginas, y La fe en Jesucristo. Ensayo desde las víctimas (1999) con 613 páginas. Pero a estas les sigue un buen número de investigaciones y escritos sobre teología, espiritualidad y sobre figuras como monseñor Romero e Ignacio Ellacuría. Entre estos menciono algunos: Liberación con Espíritu (1985), 219 páginas; El principio misericordia (1992), 267 páginas; Fuera de los pobres no hay salvación (2008), 205 páginas; Terremoto, terrorismo, barbarie y utopía (2003), 245 páginas; Monseñor Romero (1989), 215 páginas; Ignacio Ellacuría, el hombre y el cristiano (2001), 85 páginas; Cartas a Ignacio Ellacuría (2004), 154 páginas.

Bajo la dirección de ambos teólogos, y con la colaboración de muchos y muchas teólogas de América latina y de España, se ha publicado un importante compendio bastante completo de las respuestas teológicas dadas desde América Latina a las grandes realidades de fe, de la praxis individual y social, de la comunidad de los creyentes, de la sociedad, de la naturaleza y de las relaciones entre las naciones. Se trata de la obra Mysterium Liberationis. Conceptos fundamentales de la teología de la liberación (1990), 2 tomos (642 páginas y 682 páginas).

\subsection{Grandes temas de las investigaciones y publicaciones del Departamento}

Dicho esto, voy a intentar detectar las grandes temáticas y ámbitos de la Teología en las que se han centrado muchas investigaciones y publicaciones de los diferentes miembros del Departamento.

\subsubsection{A partir de la realidad}

Si el punto de partida de la Teología de la liberación es siempre el análisis de la realidad, al que nuestra universidad siempre se ha dedicado con ahínco, no debe extrañar que en las investigaciones y publicaciones de los miembros del Departamento siempre sea un referente importante la realidad: la internacional, la nacional, la política, la económica, la social y la eclesial.

\section{- Realidad social y política}

El primer gran tema en este apartado es sobre la respuesta que da nuestra teología a las realidades 
que viven nuestros pueblos y muchos colectivos dentro de ellos. Me refiero a las realidades de injusticia y opresión entre las naciones, e internamente cada una de ellas, a la exclusión y marginación que con frecuencia sufren las mayorías populares por parte de quienes tienen la riqueza y el poder, a la desigualdad de género, al irrespeto hacia la riqueza de las diferentes culturas y etnias, a las continuas violaciones de los derechos humanos más fundamentales como son la vida, la vivienda, la salud, el trabajo, la libertad y muchos otros.

De hecho, nuestra teología se ha enfrentado a dichas realidades, las ha juzgado desde la experiencia de Dios que se muestra en la Sagrada Escritura y ha impulsado una praxis liberadora. Este deseo liberador ha sido en estos últimos 30 años, y lo sigue siendo en la actualidad, como un eje transversal de toda nuestra teología. Es además el origen de muchas incomprensiones, agresiones y persecuciones sufridas.

Relacionado con el tema anterior está el de la globalización que nuestra teología ha iluminado mostrando los valores de la globalización en el amor, la misericordia, la participación de todos los países y personas en la toma de decisiones comunes y en el reparto justo de las riquezas de nuestro planeta; y mostrando también la postura de Dios ante la globalización del neocapitalismo liberal que produce desigualdad, injusticia, violencia y muerte de las personas más desfavorecidas. De esta forma, se responde a la pregunta sobre dónde está Dios en la globalización y a favor de qué globalización está Dios.

Pero nuestras investigaciones teológicas han analizado y tomado postura también ante otras realidades sociales y políticas más coyunturales y concretas, como las que afectan a nuestros países del área centroamericana, especialmente Nicaragua, Guatemala, Honduras y El Salvador. Estos análisis de las diferentes coyunturas en nuestros países se convierten en auténticos focos que iluminan desde nuestra visión teológica; la corrupción, injusticia social, desigualdad, marginación, exclusión, crimen organizado, opción por la guerra, impunidad, elecciones, participación ciudadana, Ilamada a la humanización, reconciliación, esperanza de algo nuevo y diferente, entre otros, son temas que aparecen en varios libros y artículos de miembros de nuestro Departamento como un aporte de nuestra reflexión teológica al análisis social y político.

Nuestra teología ha reflexionado también sobre la necesidad de que la política se viva de otra manera posible, no la del dominio y el poder, no como la que ejercen muy a menudo los jefes de las naciones y del poder sino la del servicio; solo así la política 
tiene futuro y puede ser factor de esperanza.

\section{- Realidad eclesial}

Nadie puede negar que para la realidad eclesial fue fundamental el Concilio Vaticano II, que abrió un nuevo camino de vivir como Iglesia en comunión, así como las historizaciones del mismo, obra de las Conferencias generales del episcopado latinoamericano, especialmente en Medellín, Puebla y Aparecida. Por eso no son extrañas algunas investigaciones de miembros del Departamento sobre el Concilio Vaticano II como fuente de esperanza en América Latina, sobre la fe como luz que ilumina la fraternidad según la Gaudium et spes y la Lumen gentium, sobre la Iglesia de los pobres que no acabó de prosperar en el Concilio a pesar del sueño de Juan XXIII pero que se historizó en la Conferencia de Medellín y que monseñor Romero hizo realidad en El Salvador.

Varias investigaciones se realizaron también sobre la preparación de la Conferencia general del episcopado en Aparecida; sus nuevos retos y tareas, sus anhelos humanos y cristianos.

Siguiendo con el episcopado latinoamericano, ya es un tópico que en Guatemala ha sido de los países donde mejor ha funcionado este episcopado porque ha sabido responder a las realidades de nuestro país hermano. A raíz de la muerte del cardenal Quezada una investigación publicada en un artículo de ECA planteó la gran responsabilidad de la Iglesia de Guatemala en dar a dicho cardenal una sucesión conveniente para que dicha Iglesia siga en la misma línea.

Un hecho eclesial muy significativo en los últimos tiempos ha sido la renuncia del papa Benedicto XVI que ha abierto la puerta al nombramiento del cardenal Bergoglio como papa Francisco. Tal como aparece en un artículo de la Revista Latinoamericana de Teología, ha sido algo que ha propiciado la renovación de la Iglesia católica y ha hecho volver a la reflexión sobre Dios y a presentar la exclusión del pobre como exclusión y rechazo de Dios, tal como se dice en una investigación sobre la Evangelii gaudium del papa Francisco.

\section{- Realidad martirial}

Dentro de la realidad eclesial, si nos centramos en nuestros países de América Latina y más en concreto en los de nuestra área geográfica, es indudable el aspecto martirial y testimonial. Si echamos una mirada a los diferentes países, prácticamente en todos encontramos grandes testigos de la fe y del compromiso por la vida digna de las personas, especialmente las más empobrecidas y excluidas, testigos que en muchos casos han acabado entregando la vida con una muerte martirial. 
Entre estas personas, por supuesto, la más sobresaliente aquí en El Salvador es la de monseñor Romero; luz y fuerza para el pueblo de El Salvador y para todos los pueblos en los momentos de máxima crisis. No es de extrañar, pues, las muchas investigaciones publicadas en forma de libros y artículos por parte de miembros de nuestro Departamento. Destaco en primer lugar la gran obra póstuma de nuestro compañero ya fallecido, Miguel Cavada, la edición crítica en seis tomos de las Homilías de Monseñor Romero a lo largo de sus tres años de ministerio como arzobispo de San Salvador.

También han sido fundamentales para mantener viva la presencia y el mensaje de monseñor Romero las recopilaciones de sus textos en el libro editado el mismo año del martirio de monseñor $y$ titulado significativamente: $\mathrm{La}$ VOZ de los sin Voz, así como algunos cuadernos de monseñor Romero como El evangelio de Monseñor Romero, El corazón de Monseñor Romero, así como varios libros y artículos en la Revista Latinoamericana de Teología y de Carta a las iglesias, y algunos otros cuadernos dedicados a su figura, su pensamiento, su pastoral, su liderazgo de la nueva Iglesia de los pobres.

Un aspecto muy importante que siempre se ha destacado de monseñor Romero es su espíritu profético, varias investigaciones y publicaciones se han centrado en este aspecto de su vida y de su actividad. En ellas aparece con claridad que monseñor Romero fue un verdadero profeta y que, al estilo de los grandes profetas de Israel, denunció los grandes ídolos que exigían como sacrificio la vida de los pobres: la religión, las potencias extranjeras, las armas, el poder y la riqueza, y anunció el Dios de la vida y la esperanza que Ilama a construir el sueño de una sociedad nueva y utópica en donde quienes la formamos nos comportemos como hermanos y hermanas, participemos en todas las decisiones, y la fundamentemos en estructuras justas y pacificadoras.

En relación a monseñor Romero, finalmente, la beatificación del mismo el 23 de mayo del año pasado ha llevado a la investigación sobre el Evangelio de Marcos y al estudio de los sumarios sobre la muerte de Jesús como iluminadores del sentido del martirio de monseñor Romero proclamado como "mártir", es decir, testigo por odio a la fe y al seguimiento de Jesús. En estos sumarios se percibe una confabulación y un hostigamiento creciente por parte de las autoridades religiosas judías contra Jesús, que desemboca en su crucifixión. El acercamiento exegético tiene como trasfondo que el papa haya declarado a monseñor Romero "Mártir por odio a la fe". Este estudio pretende descubrir claves (re)interpretativas para profundizar en esa declaración, así como 
descubrir sus implicaciones para la vida de la Iglesia.

Es muy común el paralelismo que se acostumbra a hacer de Juan Bautista como precursor de Jesús de Nazaret y de Rutilio Grande como precursor de monseñor Romero. Por esto la figura de Rutilio Grande resalta también en las investigaciones hechas por miembros del Departamento o propiciadas por el mismo Departamento. En ellas se presenta su vida y su pastoral, su capacidad y compromiso evangelizador en el área rural, las pocas homilías que se conservan de él, su relación y encuentro con monseñor Romero, y el reto de su palabra a comprometerse en favor de los pobres.

También las y los mártires del 16 de noviembre en la UCA, seguidores fieles del protomártir Jesús de Nazaret en la línea de monseñor Romero y Rutilio Grande, han sido objeto de diferentes escritos para dar a conocer sus vidas, su forma de hacerse próximos compasivamente como buenos samaritanos de este pueblo empobrecido, su espíritu fuerte, sus aspectos más humanos, su profecía y teología en favor del pueblo, su seguir vivos entre nosotros y su interpelación a seguir trabajando por la vida digna de los empobrecidos y excluidos.
En este apartado, finalmente, una figura de la Iglesia guatemalteca, monseñor Juan Gerardi, mártir por los derechos humanos, también ha sido objeto de alguna investigación en la que se ha resaltado cómo esta entrega de la vida en favor de los derechos por una vida digna produce esperanza.

\section{- Realidad y Visión y Misión de la UCA}

A partir de la opción de la Compañía de Jesús a mitad de los años 70 por el acompañamiento de una fe que lleve a promover la justicia, la UCA se ha tomado en serio su definición como universidad de inspiración cristiana por el cambio social, es decir, por la transformación de nuestras realidades en sociedades más justas, más pacíficas, más participativas.

El Departamento ha ido contribuyendo a mantener esta visión $y$ ha ido interpelando con sus reflexiones teológicas a todo el conjunto de la UCA para que siga en medio de todos como aquel que sirve, haciendo el bien y trabajando por dicha transformación social. Esta tarea el Departamento la ha realizado sobre todo a través de algunas charlas a empleados y empleadas, y ponencias en los seminarios internos en toda la universidad, ponencias que han sido publicadas en ECA o en cuadernos especiales. 


\section{- Realidad y teólogos que parten de ella}

Pero además, este interés por la realidad se muestra con claridad en las investigaciones y publicaciones de algunos miembros del Departamento de Teología, en forma de libros y artículos de la Revista Latinoamericana de Teología, sobre autores para los que la realidad es algo nuclear de su teología como pueden ser Xavier Zubiri, Juan Bautista Metz, Dietrich Bonhöffer, Karl Rahner e Ignacio Ellacuría. También, a modo de ejemplo señalo algunas temáticas respecto a ellos; en torno a la fe, el agnosticismo, la indiferencia y el ateísmo según Xabier Zubiri, la religión en Zubiri, Zubiri y la revelación del misterio de la Trinidad, la encarnación del Verbo de Dios y la creación.

Todo lo anterior hace referencia sobre la realidad. Pero el juicio sobre esta realidad, toda teología lo hace desde la reflexión humana y desde el estudio de la Sagrada Escritura para descubrir y formular de un modo inculturado lo que Dios piensa sobre esta misma realidad.

\subsubsection{Juicio de esta realidad a partir del Dios de la Biblia}

No resulta extraño, pues, que sobre todo en nuestras colecciones y en nuestra Revista Latinoamericana de Teología abunden los escritos que profundizan en la Sagrada Escritura en relación a temas de mayor actualidad.
Varias investigaciones se han centrado en la búsqueda de un eje en torno al cual giran el Antiguo y el Nuevo Testamento. El pueblo y la comunidad fueron descubriendo en su historia y en su propia vida a un Dios que siempre los acompaña y los libera de todas las esclavitudes históricas, no quiere los imperialismos de las superpotencias, comparte con la humanidad su sabiduría profundamente liberadora, rechaza la confianza en una fuerza basada en las armas, está en contra de las opresiones del poder, Ilama a una fe que no se desentienda de la justicia sino que se manifieste plenamente en las acciones en favor del bien común y especialmente en favor de los excluidos, se encarna en un Jesús que vive en la marginalidad y desde ahí hace lo necesario para que quienes han sido colocados al borde o al margen del camino se pongan a caminar con él y recobren su dignidad y sus derechos.

Todo esto se ha hecho a partir de la investigación sobre el conjunto del Antiguo y Nuevo Testamento y desde los diferentes tipos de textos: los narrativos, los legales, los sapienciales, los proféticos, los evangelios, entre otros.

Va quedando claro a lo largo de todos estos textos que Dios es el primer defensor de los derechos humanos, es un Dios con entrañas de misericordia que siempre escucha el clamor del pueblo, un 
Dios que llamó y sigue Ilamando a la reconciliación con él y con el prójimo, un Dios que espera que su pueblo, desde el sufrimiento y el dolor, luche sin cesar por eliminar el sufrimiento del mundo.

\subsubsection{Hacia una praxis y una espiritualidad liberadoras}

Pero nuestras investigaciones teológicas no se quedan en ver la realidad y juzgarla desde la palabra de Dios y la reflexión humana, sino que todas ellas llevan a la exigencia de la praxis cristiana y a la consideración de que como seres humanos somos corresponsables con Dios en que la historia de la humanidad se convierta en una historia de salvación y en que esa manera utópica y soñadora de Dios de ver la realidad se vaya haciendo realidad.

Quiero mencionar en este apartado de un modo especial toda la obra de investigación y divulgación de nuestro compañero fallecido hace cuatro años, el P. Dean Brackley. Siempre fue notoria su preocupación y su ocupación en una teología y una espiritualidad que saliesen al exterior, que se convirtieran en práctica liberadora de la realidad personal y social.

\section{Una teología que crea escuela}

Es indudable que esta teología liberadora y transformadora de la realidad desde la reflexión bíblica y dogmática ha ido creando escuela. Indicadores de esto son los diferentes trabajos de tesis de graduación de nuestros educandos de licenciatura. En ellos han profundizado en temas de la realidad vistos por los grandes teólogos analizados, teólogos que se han ocupado de dicha realidad para convertirla en algo mejor para todos y todas.
Hablando ya de los y las graduadas de nuestra maestría antigua y la actual Maestría en Teología Latinoamericana, si se repasa los temas de sus tesis de investigación, siempre han estado relacionados con temas candentes de la realidad (la justicia, la historia, la ecología, la violencia, las pandiIlas, la guerra, la paz, la reconciliación, entre otros), y con los caminos para convertir esta realidad en algo esperanzador que mira a la utopía y al sueño de Dios.

\section{Difusión de toda esta teología}

Pero toda esta investigación ¿cómo se difunde y llega a la gente?

Como ya he mencionado antes, una buena plataforma para la difusión de esta investigación y reflexión teológica ha sido, además de la docencia, las diferentes publicaciones de las que es responsable y animador nuestro Departamento. 
Paso a mencionarlas rápidamente:

Las dos colecciones de Teología Latinoamericana y La Iglesia en América Latina editadas por UCA editores.

Otros libros de miembros del Departamento o de profesores invitados muy vinculados al mismo, editados por otras editoriales internacionales, como Orbis Books, Sal Terrae, Desclée de Brouwer, Trotta, Verbo divino, entre otras.

Los numerosos artículos sobre temas relacionados con la teología liberadora aparecidos en los noventa y cinco números de la cuatrimestral Revista Latinoamericana de Teología.

Las dieciocho cartillas populares que se utilizan para las sesiones de las Escuelas de Teología Pastoral, que tratan de un modo popular de los grandes temas de la Biblia, de la Teología Sistemática y de la Práctica y pastoral

Los numerosísimos breves artículos de interpretación teológica de las realidades diarias y testimonios de las comunidades eclesiales aparecidos en los 665 números de Carta a las iglesias.

Los treinta y tres Cuadernos de Monseñor Romero aparecidos hasta el momento que tratan diversos temas bíblicos: La Biblia y el mundo de los pobres y excluidos,
El Evangelio de Judas, El Apocalipsis de Juan o el libro de la resistencia y esperanza cristiana en un mundo injusto. Los teológicos: Dios Padre, Hijo y Espíritu Santo. Los sacramentales: El Bautismo cristiano u otra manera de vivir, Eucaristía u otro mundo es posible. Los eclesiales: El Concilio Vaticano II fuente de esperanza para América Latina, En camino a Aparecida, El proyecto de Aparecida. Los testimoniales o martiriales de la Fe y de la Vida en favor del reino: Jesús el protomártir cristiano en los evangelios, Después de veinte años de Monseñor Romero, El evangelio de Monseñor Romero, El sentir con la Iglesia de Monseñor Romero, Cartas Pastorales y Discursos de Monseñor Romero, El corazón de Monseñor Romero, El Monseñor Romero de todos, Rutilio Grande y sus Homilías, XXX Aniversario del P. Rutilio Grande y su palabra comprometida con los pobres, Cinco testigos solidarias, Biografías de los Mártires de la UCA, Ignacio Ellacuría el hombre y el cristiano, Cartas de Jon Sobrino a Ignacio Ellacuría, Amando López, La santidad primordial de las víctimas, El P. Arrupe testigo y profeta, El Padre Dean. Los interpretativos de la realidad: Tsunami. Y los morales: Una sexualidad liberadora.

Quiero finalizar esta presentación con los tres congresos internacionales de Teología de la liberación, organizados hasta este momento gracias a la colaboración de nuestro Departamento y del 
Centro Monseñor Romero de la UCA, en los que han tenido sus ponencias reconocidos teólogos y teólogas de la liberación, tanto entre sus fundadores como entre sus continuadores, y en los que han participado con intervenciones impactantes entrañables, el caso de obispos como Samuel Ruiz, Pedro Casaldàliga, Álvaro Ramazzini y Raúl Vera. 